\title{
SDriver: Location-Specific Signatures Prevent SQL Injection Attacks*†
}

\author{
Dimitris Mitropoulos \\ Department of Management Science and Technology \\ Athens University of Economics and Business \\ dimitro@aueb.gr \\ Diomidis Spinellis \\ Department of Management Science and Technology \\ Athens University of Economics and Business \\ dds@aueb.gr
}

\begin{abstract}
SQL injection attacks involve the construction of application input data that will result in the execution of malicious SQL statements. Many web applications are prone to SQL injection attacks. This paper proposes a novel method for preventing this kind of attacks by placing a database driver proxy between the application and its underlying relational database management system. To detect an attack, the driver uses stripped-down SQL queries and stack traces to create SQL statement signatures that are then used to distinguish between injected and legitimate queries. The driver depends neither on the application nor on the RDBMS and can be easily retrofitted to any system. We have developed a tool, SDriver, that implements our technique and tested it successfully on several web applications.
\end{abstract}

\section{Introduction}

Traditionally, most programmers have been trained in terms of writing code that implements the required functionality without considering its many security aspects [16]. It is very common, for a programmer, to make false assumptions

${ }^{*}$ Computers and Security, 2009. DOI:10.1016/j.cose.2008.09.005

${ }^{\dagger}$ This is a machine-readable rendering of a working paper draft that led to a publication. The publication should always be cited in preference to this draft using the reference in the previous footnote. This material is presented to ensure timely dissemination of scholarly and technical work. Copyright and all rights therein are retained by authors or by other copyright holders. All persons copying this information are expected to adhere to the terms and constraints invoked by each author's copyright. In most cases, these works may not be reposted without the explicit permission of the copyright holder. 
about user input [28]. Classic examples include: assuming only numeric characters will be entered as input, or that the input will never exceed a certain length.

SQL injection attacks comprise a subset of a wide set of attacks known as code injection attacks $[18,2]$. Code injection is a technique to introduce code into a computer program or system by taking advantage of the unchecked assumptions the system makes about its inputs [31].

Many web applications have interfaces where a user can input data to interact with the application's underlying relational database management system RDBMS. This input becomes part of an SQL statement, which is then executed on the RDBMS. A code injection attack that exploits the vulnerabilities of these interfaces is called an "SQL injection attack" (SQLIA) [6, 25, 16, 27]. There are many forms of SQL injection attacks. The most common involve taking advantage of:

- incorrectly passed parameters,

- incorrectly filtered quotation characters, or

- incorrect type handling.

With this kind of attacks, a malicious user can view sensitive information, destroy or modify protected data, or even crash the entire application [1]. Consider a trivial example that takes advantage of incorrectly filtered quotation characters. In a login page, besides the user name and password input fields, there is usually a separate field where users can input their e-mail address, in case they forget their password. The statement that is probably executed can have the following form:

SELECT * FROM passwords WHERE email =

'theemaillgave@example.com';

If an attacker, inputs the string anything' $O R$ ' $x$ '=' $x$, she could conceivably view every item in the table. In a similar way, the attacker could modify the database's contents or schema.

An "incorrect type handling" attack occurs when a user-supplied field is not strongly typed or is not checked for type constraints. For example, many websites allow users, to access their older press releases. A URL for accessing the site's fifth press release could look like this [24]:

http ://www . website. com/pressRelease . jsp?RelID=5

And the statement that is probably executed is:

SELECT description, issuedate, body FROM pressRel WHERE RelID = 5

If some attackers wished to find out if the application is vulnerable to SQL injection, they could change the URL into something like:

http: //www . website.com/pressRelease. jsp?pressReleaseID=5\%20AND $\% 201=1$ 


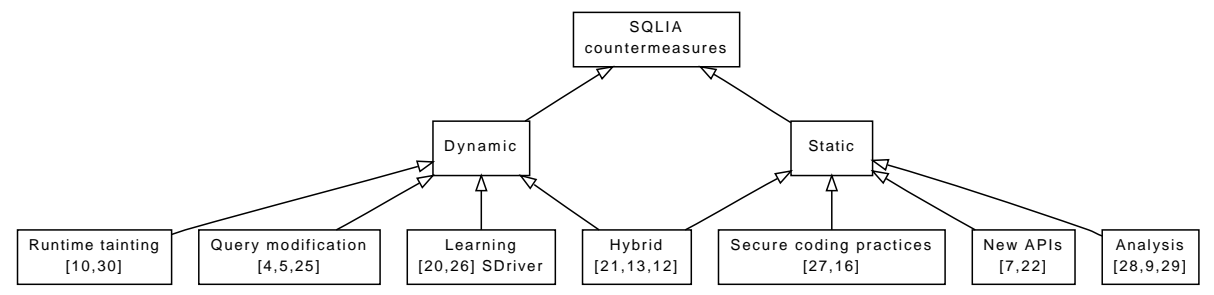

Figure 1: A taxonomy of SQLIA countermeasures

If the page displayed is the same page as before, it is clear that the field RelID is not strongly typed and end users can manipulate the statement as they choose. Note that, while the first attack could be countered by filtering out the quotation characters from the input data, countering the second attack would require code to ensure that the input data is a single integer. According to vulnerability databases like CVE (Common Vulnerabilities and Exposures) ${ }^{1}$ SQLIA incidents have increased significantly over the last years.

\section{Countering SQL Injection Attacks}

A taxonomy of SQLIA countermeasures appears in Figure 1. Static approaches detect or counteract the possibility of an SQLIA at compile time, while dynamic approaches perform this task at runtime. Note that both approaches may require the analysis or modification of an application's source code.

On the static front an often repeated mantra is the adoption of secure coding practices $[27,16]$. Most SQL injection attacks can be prevented by passing user data as parameters of previously prepared SQL statements instead of intermingling user data directly in the SQL statement. For example, the statement we examined previously, could be passed to the database with a question mark used as a placeholder for the parameter, and a separate type-checked API call could be used for setting the first parameter of the SQL statement to the desired value. In Java, this would be accomplished by the following method calls.

PreparedStatement pstmt $=$ con.prepareStatement (

"SELECT description, issuedate, body FROM pressRel" +

"WHERE RelID = ?");

pstmt.setInt (1, 5);

These practices can indeed increase the robustness of applications. However, experience has shown us that the expectation for them to be embraced to the extent of completely eliminating security vulnerabilities is just wishful thinking.

An alternative approach involves the introduction of type-safe programming interfaces, like DOM SQL [22] and the Safe Query Objects [7]. Both

${ }^{1}$ http://cve.mitre.org/ 
eliminate the incestuous relationship between untyped Java strings and SQL statements, but don't address legacy code, while also requiring programmers to learn a radically new API.

An approach that deals with existing code and coding practices involves the static analysis of the application's source code to locate SQL statement invocations that are considered unsafe $[28,9,29]$. While the impact of tools based on these methods on development and deployment processes is minimal, their accuracy and scope is reduced by the complexity of modern web-based application frameworks. For instance, recent work by Wassermann and Su [29] proposes a sound and precise approach, which however depends on specifying the semantincs of all PHP string functions. (The implementation described contains specifications for 243 functions.)

On the dynamic front runtime tainting approaches enforce security policies by marking untrusted data and tracing its flow through the program. For instance the system by $\mathrm{Xu}$ et al. [10] covers applications whose source code or their interpreter is written in C, while the work by Haldar et al. [30] targets Java code. These approaches generally require significant changes to a language's compiler or its runtime system.

Another dynamic approach involves query modification. Here the modified query is either reconstructed at runtime using a cryptographic key that is inaccessible to the attacker [4], or the user input is tagged with delimiters that allow an augmented SQL grammar to detect SQLIAs [5, 25]. Both approaches require significant source code modifications.

Training approaches are based on the ideas of Denning's original intrusion detection framework [8]: they record and store valid SQL statements and thereby detect SQLIAS as outliers from the set of valid statements. An early approach, DIDAFIT [20] recorded all database transactions. Subsequent refinements tagged each transaction with the corresponding application [26]. Our system further improves on these techniques by automatically determining precisely each query's location through its stack trace.

Finally, some approaches combine a static analysis with runtime monitoring. For instance, AMNESIA, associates a query model with the location of each query in the application and then monitors the application to detect when queries diverge from the expected model [13, 12]. A more general hybrid approach involves the location of SQLIAs using the program query language PQL [21]. The PQL queries are evaluated through both a static analysis and the dynamic monitoring of instrumented code. These approaches, although complex to implement, seem to offer an additional margin of protection against false positives and negatives.

Readers looking for a more detailed survey of SQL injection attacks and the corresponding countermeasures can turn to the recently published survey by Halfond and his colleagues [14].

In this paper we propose a novel technique of preventing SQLIAs. Our technique incorporates a driver that stands between the web front-end and the backend database. The key property of this driver is that every SQL statement can be identified using the query's location and a stripped-down version of its contents. By analyzing these characteristics during a training phase, we can build a 


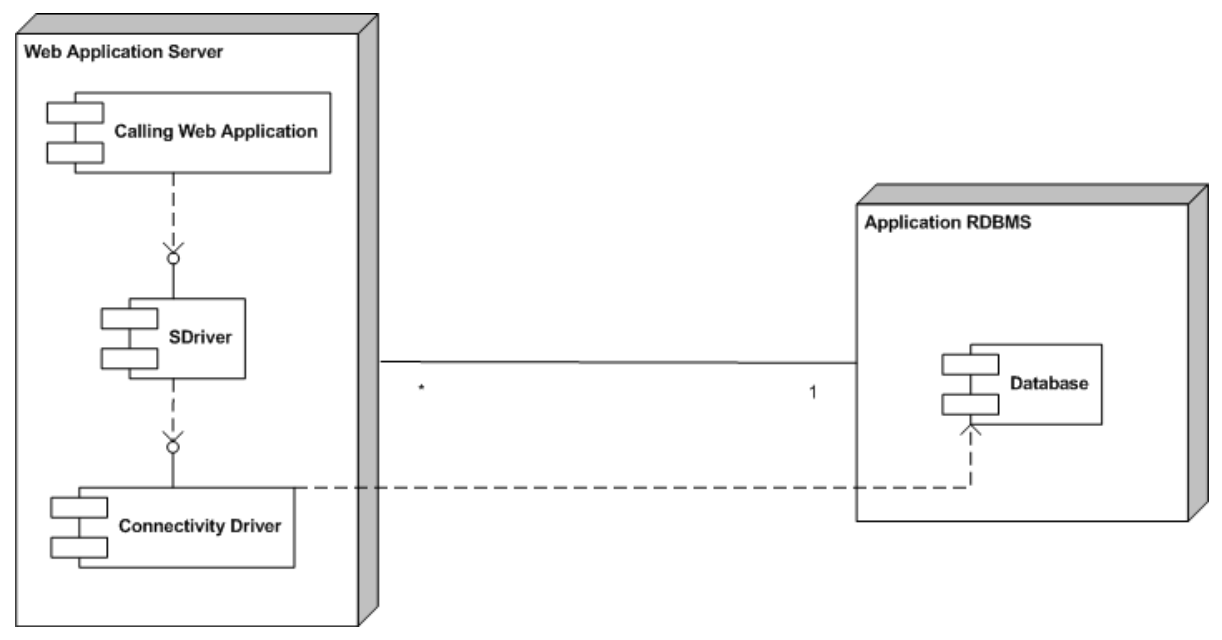

Figure 2: The architecture of our proposed driver

model of the legitimate queries. ${ }^{2}$ Then at runtime our driver checks all queries for compliance with the trained model and can thus block queries containing additional maliciously injected elements. The work reported here builds upon an earlier prototype [23] with a more robust SQL processing technique, significant performance improvements, and more extensive validation experiments.

\section{A Signature-Based Proxy Driver}

The architecture of typical tiered web applications consists of at least an application running on a web server and a back-end database [28]. Between these two tiers, there is in most cases a database connectivity driver based on protocols like ODBC (Open Database Connectivity) or JDBC (Java Database Connectivity). The main function of such a driver is to provide a portability layer by obtaining SQL statements from the application and forwarding them to the database. The driver that we propose is also a connectivity driver that operates however as a shim or proxy standing between the application and the database interface driver (see Figure 2). Our driver is transparent: its only role is to prevent SQLIA, and it depends neither on the application, nor on the underlying connectivity driver.

To work as a connectivity driver, our driver implements the complete interface of the connectivity protocol. However, most of the driver's methods simply forward the request to the underlying connectivity driver. Only a few methods capture and process requests in order to prevent SQLIAS. In this respect our

\footnotetext{
${ }^{2}$ From now on we will often use the term "query" to denote all SQL statements. Although SQL data manipulation, definition, and control statements are not queries, using the term "query" avoids confusion between the SQL statements and the statements of the general purpose programming language where SQL elements are often embedded.
} 
driver acts as a proxy for the underlying driver working as a firewall between the original driver and the application.

In order to secure the application from SQLIAs the driver must go through a training phase. This involves executing all the SQL queries of the application so that the driver can identify them in a way we will show in the next section. Then, the driver's operation can shift into production mode, where the driver takes into account all the trained legitimate queries to prevent SQLIAs by detecting and blocking them.

\subsection{Training Mode}

Every SQL query of an application is identified through a signature created by combining three of its characteristics.

1. The method invocation stack trace. This includes the details of all methods and call location, from the method of the application where the query is executed down to the target method of the connectivity driver.

2. The SQL keywords.

3. The tables and the fields that the query uses in order to retrieve its results.

By combining all the fixed elements of each query with its invocation method's stack trace, we obtain a unique identifier - signature - for all the legitimate queries of an application. A formal representation of the application's signatures that should be accepted as legitimate is the following: If during an application's normal (non-attacked) run, $K$ is the set of method stack traces at the point where an SQL statement is executed; $L$ is the set of the corresponding SQL keyword names; $M$ the set of the corresponding database table names, and $N$ the set of the corresponding table field names, the set of the legitimate query signatures $S$ is defined as follows:

$$
S=\left\{\omega: \omega=\left(k, a_{1}, a_{2}, \ldots\right), k \in K, a_{i} \in(L \cup M \cup N)\right\}
$$

When the system operates in the training mode, each query signature $Q$ is added to $S$. In production mode a query with a signature $Q$ is considered legal iff $Q \in S$. Obviously, a query cannot be unambiguously identified by using just one of the above characteristics, such as the query's keywords, and this is why all of them are combined into a tuple.

To combine these characteristics, when a query is being sent to the database our driver carries out two actions. First, it strips down the query, removing all numbers and string literals. So if the following statement is being executed

SELECT table1.field1 FROM table1 WHERE table1.field2 = 'foo' AND table1. field $3>3$

the driver removes 'foo' and 3 from the query string.

The driver also traverses down the call stack, saving the details of each method invocation, until it reaches the statement's origins. The association of 
stack frame data with each SQL query - a process that to the best of our knowledge is unique to our approach - is an important defense against maliciously crafted attacks that try to masquerade as legitimate queries.

As an example, consider an application that will send the password for a forgetful user, Alice, via email by executing

SELECT password from userdata WHERE id = 'Alice'

This same application could allow users to lock their terminal, but allow the unlocking either with the user's password or with the administrator password (the 4.3 BSD lock command behaved in this peculiar way). The corresponding query to verify the password on the locked Alice's workstation would be as follows.

SELECT password from userdata WHERE id = 'Alice' OR id = 'admin'

It is now easy to see that a malicious user, Bob, could obtain the administrator's password by email by entering on the password retrieval form as his user identifier

nosuchuser' OR id = 'admin

Without the differentiating factor of a stack trace, the preceding query would have the same signature as the one used for unlocking the terminal, and would therefore escape a traditional signature-based SQLIA protection system.

Our initial design had sDriver storing each query's keywords, table names, and stack trace into separate tables of an auxiliary database. During implementation we realized that, because the only operations we were interested in were adding a query $Q$ to the set of known queries $S$ and testing whether $Q \in S$, we could substitute the full signature $S$ with its hash. This substitution is valid, because sDriver operates under the premise of best effort rather than absolute correctness [15]. Therefore, the stack trace and the stripped down query are concatenated and the driver applies a hash function on them to form the stored form of the query signature. When the system is operating in training mode, all the signatures are saved in an auxiliary database table, so that when the system operates in production mode the driver can check whether a query is legitimate or not. This is done off-line since an on-line training could lead to disputable signatures. Specifically, if an attack is attempted the generated signature is going to be stored as a legitimate one putting the system's operation at risk.

\subsection{Production Mode}

The driver's functionality during the production mode does not differ significantly from the one in the training mode. The steps are the same until the driver derives the query's signature. At that point, the driver consults the database table of saved query signatures to verify that the query is legal. This interaction though, happens in an indirect way as we describe in Section 5.2. If the driver identifies it as a legitimate one then the query passes through. If it does not, then the application is probably under attack. In such a case the driver can 


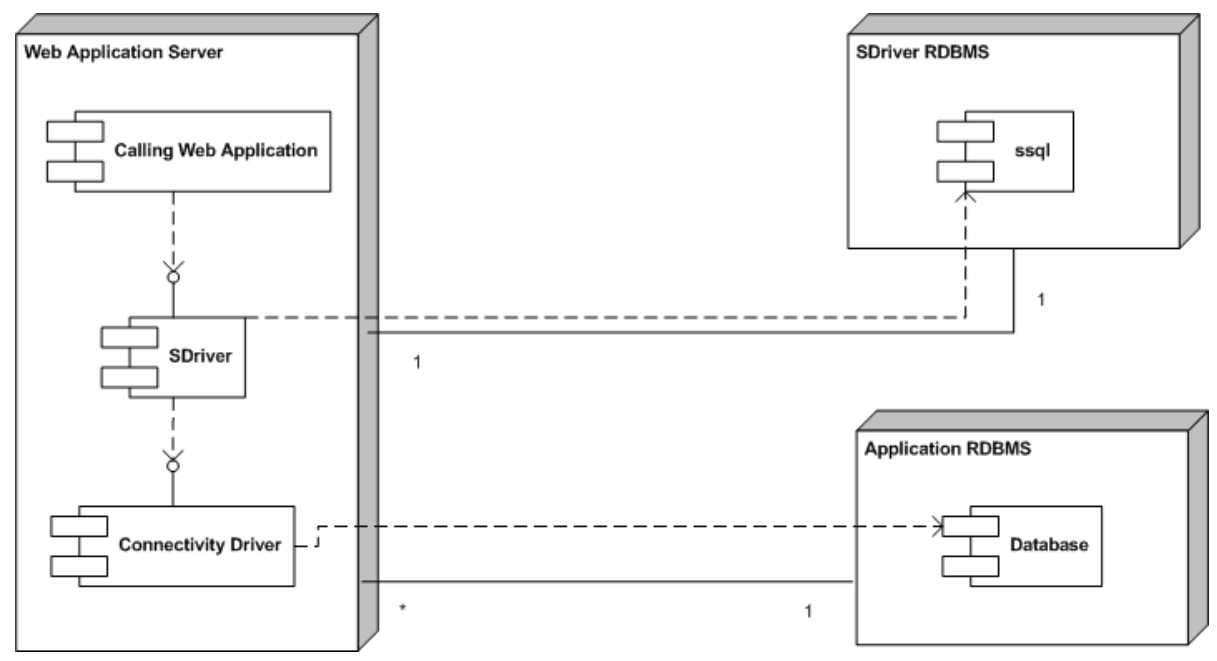

Figure 3: sDriver's architecture

halt the application with an exception, it can log an error message, or it can forward an alarm to an enterprise-wide intrusion detection system.

As an example, consider an attack that takes advantage of incorrectly filtered quotation characters. The additional keywords that the malicious user injects will definitely lead to an unknown signature. In this case the driver becomes aware of the attack and prevents it.

\section{Java Platform Implementation}

We have implemented our solution in the Java platform, but implementations in other operating environments are certainly feasible. The secure database driver, which we call sDriver, acts as a JDBC driver wrapped around other drivers that implement a database's JDBC protocol (see Figure 3).

\subsection{Proxy Interface}

JDBC drivers known as "native-protocol drivers" 3 (or type 4 JDBC drivers) convert JDBC calls directly into the vendor-specific database protocol. At the client's (application) side, a separate driver is needed for each database. sDriver does not depend on the application or the native driver and it is placed between them. To accomplish that, the application must be modified in a single position: in the location where the application establishes a connection with a driver. For the application to be secured, the sDriver must establish a connection with the driver that the application is meant to use. To achieve that, we pass the driver's name through the URL of the original connection (see Figure 3). For example, if

${ }^{3}$ http://java.sun.com/products/jdbc/driverdesc.html 


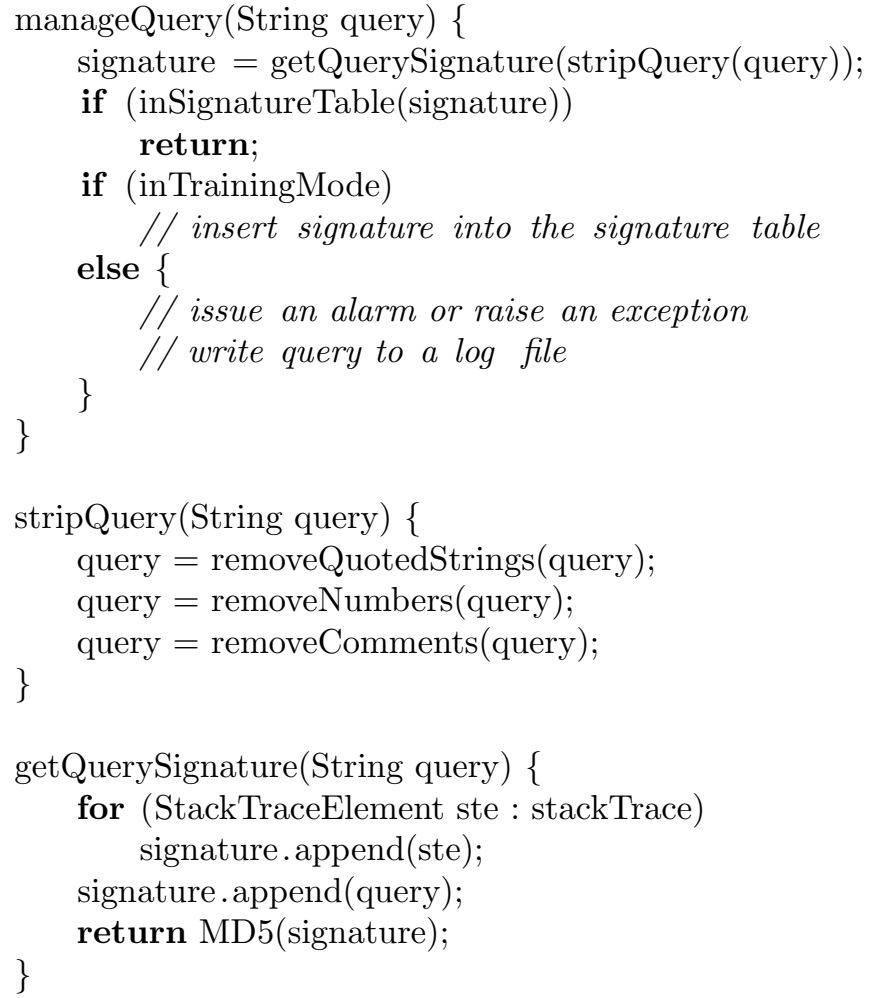

Figure 4: The operation of sDriver

the application is meant to connect to the Microsoft SQL Server 2000 the source code would look like this:

Class.forName ("com.microsoft.jdbc.sqlserver.SQLServerDriver"); Connection conn=DriverManager.getConnection( "jdbc:microsoft: sqlserver :/ / localhost :1433; databasename=MyDB",

"username", "password");

The modified code for using the sDriver would be:

Class.forName ("org.SDriver");

Connection conn $=$ DriverManager.getConnection ( "jdbc:com.microsoft.jdbc. sqlserver .SQLServerDriver:" +

"microsoft: sqlserver :// localhost :1433; databasename = MyDB", "username", "password"); 


\subsection{Implementation Details}

SDriver is not a classic native-protocol RDBMS driver. The implementation of most of the driver's methods simply involves calling the corresponding methods of the underlying driver. Only a few methods from those that a native-protocol driver implements pass SQL code through them, and can therefore be used to launch an SQLIA. These methods are the various forms of addBatch, execute, executeQuery, and executeUpdate. To secure applications against SQLIAs, SDriver interposes itself in these methods examining the query string that is about to be executed. For this examination to take place, sDriver follows the steps of the pseudocode listed in Figure 4.

Figure 3 shows that sDriver depends on another database component called $s s q l$. This works as the signature data store.

One of the tricky parts of the sDriver implementation is the code that traverses the application's stack. Perversely, in Java the way to access the stack frames is to create an object of type Throwable. The class Throwable is the superclass of all errors and exceptions in Java. To aid the display and debugging of exceptions Throwable objects support a method called getStackTrace, which returns an array of stack frames. Each stack frame provides methods for obtaining the corresponding file name, method name, and line number. The following listing shows the contents of a stored stack trace:

com.SStatement.getQuerySignature (SStatement.java:556)

com.SStatement.manageQuery (SStatement. java:489)

com.SStatement. executeQuery (SStatement. java:430)

beans.querybean. selection1 (querybean. java:20)...

Every stack element contains information about a method invocation including the method name, the package, the file, and the line number. The first method will always be getQuerySignature because it is the one that traverses the call stack. The element that participates more in the diversity of a signature is typically the fourth one: the application's method that directs the connectivity driver to pass the query to the database.

\section{Evaluation}

The success of any system claiming to improve security typically depends on the accuracy of its results (often measured in terms of false positives and negatives) and on its cost in terms of deployment, operation, and maintenance.

\subsection{Accuracy}

We evaluated the accuracy of sDriver through three experiments: a synthetic benchmark, a notoriously insecure application, and a bundle of previously evaluated real-world applications.

Our synthetic benchmark was a JSP application with the same technical characteristics as those described in reference [4]. This application allowed a 
user to inject SQL into a "where" clause with no input validation, and retrieve information concerning application data. After placing sDriver between the application and the database, the attack was successfully prevented.

We then searched for a real-world web application that had a record of being vulnerable to SQLIAS. According to the common vulnerability database CVE and the security bulletin providers US-CERT, ${ }^{4}$ Secunia, ${ }^{5}$ and Armorize Technologies, ${ }^{6}$ a notoriously vulnerable application is Daffodil CRM $1.5 .^{7}$ In Daffodil 1.5, remote attackers could execute arbitrary SQL commands via unspecified parameters in a login action. In particular, users wanting to access Daffodil had to fill-in a simple username and password form. By using a SQLIA similar to the one we presented in Section 1, an unauthorized user could access administrator facilities. SDriver recognized and blocked the attack, without otherwise interfering with Daffodil's operation.

Finally, we selected five real-world web applications that have been used in the literature for previous evaluations ${ }^{8}[11,25]$. We attempted a wide variety of attacks based on incorrectly filtered quotation characters, incorrectly passed parameters, untyped parameters, tautologies, and others $[1,14]$.

Table 1 shows, for each web application, the number of the signatures stored in the sDriver database after training, the number of unsuccessful attacks (attacks that did not get past the application's defenses), the number of successful attacks (attacks that could potentially compromise the application), and the number of attacks prevented by sDriver, in absolute terms and as a percentage over the total number of successful attacks. The table's columns follow the labeling introduced in reference [11].

\begin{tabular}{lrrrr}
\hline Application & Signatures & Unsuccessful & Successful & Prevented \\
\hline synthetic benchmark & 21 & 55 & 29 & $29(100 \%)$ \\
daffodil & 72 & 77 & 27 & $27(100 \%)$ \\
bookstore & 168 & 288 & 39 & $39(100 \%)$ \\
classifieds & 122 & 270 & 37 & $37(100 \%)$ \\
employee directory & 61 & 207 & 31 & $31(100 \%)$ \\
events & 65 & 115 & 29 & $29(100 \%)$ \\
portal & 156 & 312 & 49 & $49(100 \%)$ \\
\hline
\end{tabular}

Table 1: sDriver's Precision

While testing, we realized that the five previously evaluated applications shared a common feature: in a misguided attempt to avoid SQLIAs, they scanned user input for single quotation marks and replaced them with double quotation marks. This technique masked the SQLIA problem, but introduced a data leakage

\footnotetext{
${ }^{4}$ http: //www.us-cert.gov/

${ }^{5}$ http://secunia.com

${ }^{6}$ http://www . armorize.com

${ }^{7}$ Daffodil can be obtained from http://www.daffodildb.com/crm/

${ }^{8}$ The applications can be obtained from http://www.gotocode.com/
} 


\begin{tabular}{lrrr}
\hline Application & \multicolumn{3}{c}{ Execution time (ns) } \\
Database & Original & SDriver & Overhead (\%) \\
\hline SQL Server & 175 & 183 & 4.7 \\
MySQL & 121 & 126 & 3.7 \\
\hline
\end{tabular}

Table 2: Proxy driver baseline cost

\begin{tabular}{lrrrrr}
\hline Application & \multicolumn{3}{c}{ Execution time $(\mu \mathrm{s})$} & \multicolumn{2}{c}{ Overhead $(\%)$} \\
Database & Original & Training & Production & Training & Production \\
\hline SQL Server & 605 & 1221 & 841 & 102 & 39 \\
MysQL & 401 & 1009 & 613 & 60 & 35 \\
\hline
\end{tabular}

Table 3: The cost of SQL query processing under SDdriver

vulnerability. For instance, a user input parameter consisting of the string any ' $^{\prime}$ in the application "portal" would result in the execution of the following query:

SELECT e.date_start AS e_date_start, e.event_desc AS e_event_desc,

e.event_name $\mathbf{A S}$ e_event_name, e.location $\mathbf{A S}$ e_location,

e.presenter AS e_presenter FROM events e WHERE

(e.event_desc LIKE '\%any "\%' OR e.event_name LIKE '\%any $\ " \%$ ' OR

e.presenter LIKE '\%any "\%') ORDER BY e.date_start DESC

The preceding statement would raise an exception revealing information about the underlying database and its schema.

Given that our driver works as a wrapper around other connectivity drivers, we could also instrument it to handle exceptions when running in production mode. As a result, critical information like the above would not be revealed. However, because well-written applications have their own sophisticated exception handling, we made secure exception handling an optional configurable feature.

With its secure exception handling activated, our tool successfully prevented all SQLIAs in this last test without suffering from false negatives. Furthermore, we did not encounter false positives (legitimate queries misreported as an attack) in any of the three experiment classes we performed.

\subsection{Operation Cost}

The acquisition cost of sDriver is minimal, because we are releasing it as opensource software. ${ }^{9}$ Deploying sDriver is also relatively straightforward: the only requirement is the ability to modify the database's connection string. This can be achieved by specifying an appropriate application-specific parameter (Java property), by modifying the source code, or (in extreme cases) by patching the application's binary. Furthermore, one must then execute the application

\footnotetext{
${ }^{9}$ The software is available at istlab.dmst.aueb.gr/ dimitro/sdriver
} 
in training mode. An automated test suite that will exercise (ideally all) the application's calls to methods containing SQL strings with user-input data, would make this exercise trivial. Otherwise appropriate scenarios must be devised and executed each time a new version of the application is installed.

The driver's architecture allowed us to test its performance on two RDBMSs: SQL Server 2000 and MysqL (version 5.0.24). All tests were performed on a Pentium $4 \mathrm{CPU}$ clocked at $2.6 \mathrm{GHz}$ on a machine with 512MB RAM running Java 1.6.0 under Windows XP Professional. We first measured the baseline overhead of sDriver by executing a JDBC method - getAutoCommit() - that is passed through directly to the underlying database driver without further processing. The results, appearing in Table 2 indicate that the cost of interposing SDriver is negligible.

Subsequently, we measured the overhead of the SQLIA detection code by executing the following moderately complex SQL statement, with and without SDriver.

SELECT d_name, d_SorL, d_year,d_genre, d_cover FROM artists, disks, recorded WHERE a_name = "'+ selectedartist +"' AND

$\mathrm{d} \_n a m e=$ rec_d_name $\mathbf{A N D}$ rec_a_name $=$ a_name

The performance overhead for the two RDBMSs was similar (see Table 3). In training mode the queries take twice as long to execute. However, this cost is not unreasonable, because this is an execution mode that will be rarely exercised. In production mode, the operation cost is significantly lower: below $50 \%$ for both RDBMSs. Early versions of our tool, incurred a significant overhead (with a range from $239 \%$ to $279 \%$ in both training and production mode). We optimized away this overhead by streamlining the regular expressions used for stripping the SQL queries, and by caching the signatures into a static Hashtable when a connection between the application and the sDriver is first set up in production mode. We also considered limiting the depth of the stack frame processing during production mode, by calculating in training mode a stack frame prefix tree (trie) [19, 481-490], but the performance improvements were negligible.

\section{Conclusions}

SDriver is a mechanism and a prototype application that prevents SQLIAs against web applications. If an SQL injection happens, the structure of the query, and therefore its signature will be altered, and sDriver will be able to detect it. By associating a complete stack trace with the root of each query, sDriver can correlate queries with their call sites. This increases the specificity of the stored query signatures and avoids false negative results. The increased specificity of the signatures also allows us to discard a large number of the query's elements, thereby also reducing false positive results. A disadvantage of our approach is that when the application is altered, the new source code structure invalidates existing query signatures. This necessitates a new training phase. However, with the increased adoption of test-driven development [17], and use of automated 
testing frameworks, like JUnit [3], this training phase can often become part of the application's testing.

The main contribution of our approach is the association of complete stack traces with each query. Although we have implemented sDriver as a JDBC proxy, the same approach could also be used for applications written in other languages, like $\mathrm{C}$ and $\mathrm{C}++$. Furthermore, the association of queries with their stack trace can be used to minimize the extent of source code modification in other approaches, like AMNESIA [13, 12].

Future work on our system involves packaging it in a way that will allow its straightforward deployment, and experimentation with different approaches for handling the reported attacks.

\section{References}

[1] C. Anley. Advanced SQL Injection in SQL Server Applications. Next Generation Security Software Ltd., 2002.

[2] E. Barrantes, D. Ackley, S. Forrest, T. Palmer, D. Stefanovic, and D. Zovi. Randomized instruction set emulation to disrupt binary code injection attacks. In CCS 2003: Proceedings of the 10th ACM Conference on Computer and Communications Security, pages 281-289, October 2003.

[3] Kent Beck and Erich Gamma. Test infected: Programmers love writing tests. Java Report, 3(7):37-50, July 1998.

[4] S. Boyd and A. Keromytis. SQLrand: Preventing SQL injection attacks. In M. Jakobsson, M. Yung, and J. Zhou, editors, Proceedings of the 2nd Applied Cryptography and Network Security (ACNS) Conference, pages 292304. Springer-Verlag, 2004. Lecture Notes in Computer Science Volume 3089 .

[5] G. Buehrer, B.W. Weide, and P.A. Sivilotti. Using parse tree validation to prevent SQL injection attacks. In Proceedings of the 5th international Workshop on Software Engineering and Middleware, pages 106-113. ACM Press, September 2005.

[6] CERT. CERT vulnerability note VU282403. Online http://www . kb . cert . org/vuls/id/282403, 2002. Accessed, January 7th, 2007.

[7] W.R. Cook and S. Rai. Safe query objects: statically typed objects as remotely executable queries. In ICSE 2005: 27th International Conference on Software Engineering, pages 97-106, 2005.

[8] Dorothy Elizabeth Robling Denning. An intrusion detection model. IEEE Transactions on Software Engineering, 13(2):222-232, February 1987. 
[9] Carl Gould, Zhendong Su, and Premkumar Devanbu. Static checking of dynamically generated queries in database applications. In ICSE '04: Proceedings of the 26th International Conference on Software Engineering, pages 645-654, Washington, DC, USA, 2004. IEEE Computer Society.

[10] Vivek Haldar, Deepak Chandra, and Michael Franz. Dynamic taint propagation for Java. In ACSAC '05: Proceedings of the 21st Annual Computer Security Applications Conference, pages 303-311, Washington, DC, USA, 2005. IEEE Computer Society.

[11] W. G. Halfond and A. Orso. AMNESIA: analysis and monitoring for neutralizing SQL-injection attacks. In Proceedings of the 20th IEEE/ACM international Conference on Automated Software Engineering, pages 174183. ACM Press, November 2005.

[12] W. G. Halfond and A. Orso. Preventing SQL injection attacks using AMNESIA. In ICSE 2006: Proceedings of the 28th International Conference on Software Engineering, pages 795-798. ACM Press, May 2006.

[13] William G. J. Halfond and Alessandro Orso. Combining static analysis and runtime monitoring to counter SQL-injection attacks. In WODA '05: Proceedings of the Third International Workshop on Dynamic Analysis, pages 1-7, New York, NY, USA, 2005. ACM Press.

[14] William G.J. Halfond, Jeremy Viegas, and Alessandro Orso. A classification of SQL-injection attacks and countermeasures. In Proceedings of the International Symposium on Secure Software Engineering, March 2006.

[15] Val Henson. An analysis of compare-by-hash. In Proceedings of HotOS IX: The 9th Workshop on Hot Topics in Operating Systems, pages 13-18, Berkeley, CA, May 2003. USENIX Association.

[16] Michael Howard and David LeBlanc. Writing Secure Code. Microsoft Press, Redmond, WA, second edition, 2003.

[17] Ron Jeffries and Grigori Melnik. Guest editors' introduction: TDD-the art of fearless programming. IEEE Software, 24(3):24-30, May 2007.

[18] G. S. Kc, A. D. Keromytis, and V. Prevelakis. Countering code-injection attacks with instruction-set randomization. In CCS 2003: Proceedings of the 10th ACM Conference on Computer and Communications Security, pages 272-280. ACM Press, October 2003.

[19] Donald E. Knuth. The Art of Computer Programming, volume 3: Sorting and Searching. Addison-Wesley, Reading, MA, 1973.

[20] Sin Yeung Lee, Wai Lup Low, and Pei Yuen Wong. Learning fingerprints for a database intrusion detection system. In Dieter Gollmann, Günter Karjoth, and Michael Waidner, editors, ESORICS '02: Proceedings of the 7th European Symposium on Research in Computer Security, pages 264-280, 
London, UK, 2002. Springer-Verlag. Lecture Notes In Computer Science 2502 .

[21] Michael Martin, Benjamin Livshits, and Monica S. Lam. Finding application errors and security flaws using PQL: a program query language. In OOPSLA '05: Proceedings of the 20th Annual ACM SIGPLAN Conference on Object Oriented Programming, Systems, Languages, and Applications, pages 365-383, New York, NY, USA, 2005. ACM Press.

[22] Russell A. McClure and Ingolf H. Krüger. SQL DOM: Compile time checking of dynamic SQL statements. In ICSE '05: Proceedings of the 27th International Conference on Software Engineering, pages 88-96, 2005.

[23] Dimitris Mitropoulos and Diomidis Spinellis. Countering SQL injection attacks with a database driver. In Theodore S. Papatheodorou, Dimitris N. Christodoulakis, and Nikitas N. Karanikolas, editors, Current Trends in Informatics: 11th Panhellenic Conference on Informatics, PCI 200\%, volume B, pages 105-115, Athens, May 2007. New Technologies Publications.

[24] K. Spett. Blind SQL injection. Online http://www.spidynamics.com/ whitepapers/Blind_SQLInjection.pdf, 2004. Accessed, January 7th, 2007.

[25] Zhendong Su and Gary Wassermann. The essence of command injection attacks in web applications. In Conference Record of the 33rd ACM SIGPLAN-SIGACT Symposium on Principles of Programming Languages POPL '06, pages 372-382. ACM Press, January 2006.

[26] Fredrik Valeur, Darren Mutz, and Giovanni Vigna. A learning-based approach to the detection of SQL attacks. In Klaus Julisch and Christopher Kruegel, editors, Intrusion and Malware Detection and Vulnerability Assessment: Second International Conference, DIMVA 2005, pages 123-140, July 2005. Lecture Notes in Computer Science 3548.

[27] John Viega and Gary McGraw. Building Secure Software: How to Avoid Security Problems the Right Way. Addison-Wesley, Boston, MA, 2001.

[28] Gary Wassermann and Zhendong Su. An analysis framework for security in web applications. In SAVCBS 2004: Proceedings of the FSE Workshop on Specification and Verification of Component-Based Systems, pages 70-78, 2004.

[29] Gary Wassermann and Zhendong Su. Sound and precise analysis of web applications for injection vulnerabilities. In PLDI '07: Proceedings of the 2007 ACM SIGPLAN conference on Programming language design and implementation, pages 32-41, New York, NY, USA, 2007. ACM Press.

[30] Wei Xu, Sandeep Bhatkar, and R. Sekar. Taint-enhanced policy enforcement: A practical approach to defeat a wide range of attacks. In Security 
'06: Proceedings of the 15th USENIX Security Symposium, pages 121-136, Berkeley, CA, August 2006. USENIX Association.

[31] Yves Younan, Wouter Joosen, and Frank Piessens. A methodology for designing countermeasures against current and future code injection attacks. In IWIA 2005: Proceedings of the Third IEEE International Information Assurance Workshop, College Park, Maryland, U.S.A., March 2005. IEEE, IEEE Press.

Id: sqlia.tex,v 1.61 2008/10/13 13:32:18 dimitro Exp 